\title{
Ovarian Pregnancy
}

National Cancer Institute

\section{Source}

National Cancer Institute. Ovarian Pregnancy. NCI Thesaurus. Code C92945.

An abnormal pregnancy in which the conception is implanted on the ovary. 